\title{
Multicomponent Diffusion of Lysozyme in Aqueous Calcium Chloride. The Role of Common-Ion Effects and Protein-Salt Preferential Interactions
}

\author{
Onofrio Annunziata, ${ }^{*}, \dagger$ Luigi Paduano, ${ }^{\dagger, \dagger}$ and John G. Albright ${ }^{\dagger}$ \\ Department of Chemistry, Texas Christian University, Fort Worth, Texas 76129 and Dipartimento di Chimica, \\ Università di Napoli Federico II, Naples, 80126 Italy
}

Received: March 27, 2007; In Final Form: June 14, 2007

To investigate the effect of calcium salts on the thermodynamic and transport properties of aqueous solutions of proteins, we report ternary diffusion coefficients for the lysozyme- $\mathrm{CaCl}_{2}-$ water ternary system at $25^{\circ} \mathrm{C}$ and $\mathrm{pH}$ 4.5. We have used our ternary diffusion coefficients to calculate preferential-interaction coefficients as a function of salt concentration. This has allowed us to characterize protein-salt thermodynamic interactions. We have observed the presence of large common-ion effects by examining the dependence of the diffusion coefficients on salt concentration. Our results are compared to those previously reported for the lysozyme$\mathrm{MgCl}_{2}$-water ternary system. We have found that the common-ion effect is essentially the same for both salt cases. On the other hand, by examining the dependence of the preferential-interaction coefficient on salt concentration, we have found that salt preferentially interacts with the protein in the lysozyme $-\mathrm{CaCl}_{2}-$ water system, whereas water preferentially interacts with the protein in lysozyme $-\mathrm{MgCl}_{2}-$ water system. This is consistent with the known generally larger affinity of $\mathrm{Mg}^{2+}$ for water, as compared to $\mathrm{Ca}^{2+}$, and the different roles that these two divalent metal ions play in biochemical processes. We remark that neglecting the common-ion contribution of the preferential-interaction coefficient can lead to qualitatively inaccurate descriptions of protein-salt aqueous systems, even at high salt concentrations. Indeed, for the lysozyme$\mathrm{CaCl}_{2}$ system, this approximation would lead to interpretations inconsistent with the known destabilizing effect of calcium ions on proteins.

\section{Introduction}

The chemical potential of proteins in aqueous solution is perturbed by the presence of osmolytes, such as salts, nonionic polymers, sugars, denaturants, and other small organic molecules. ${ }^{1-4}$ The primary thermodynamic effects of these additives are known to be protein preferential hydration, protein binding, molecular crowding, and Donnan equilibrium. Understanding how the protein chemical potential is affected by the concentration and nature of osmolytes is a necessary step for elucidating the mechanism of interaction between protein and osmolyte and for osmolyte applications to modulating protein unfolding, protein-ligand binding, protein solubility, protein self-assembly, and enzyme catalysis. ${ }^{1-10}$

Protein-osmolyte preferential-interaction coefficients, which are thermodynamically linked to the dependence of the protein chemical potential on osmolyte concentration, are the primary thermodynamic parameters used to characterize protein-osmolyte interactions. ${ }^{11}$ These coefficients have been interpreted in terms of models based on the existence of two domains. ${ }^{12}$ The first domain is represented by the water-osmolyte layers surrounding the protein macromolecules. This local domain is in chemical equilibrium with a bulk domain, representing the water-osmolyte remaining solution. Since proteins interact with the osmolyte and water molecules in their vicinity, the concentration of osmolyte in the local domain is different from that of the unperturbed bulk domain. If the osmolyte concentration in

* To whom correspondence should be addressed: Phone: (817) 2576215. Fax: (817) 257-5851. E-mail: O.Annunziata@tcu.edu.

Texas Christian University.

† Università di Napoli Federico II the local domain is lower than that of the bulk domain, protein preferential hydration occurs. In this case, the preferentialinteraction coefficient is negative. On the other hand, a positive value of this coefficient is obtained if the protein preferentially interacts with the osmolyte. Osmolytes that preferentially interact with proteins are also known to destabilize the protein native structure and promote unfolding. ${ }^{13}$

Among all osmolytes, inorganic salts have been extensively used in several biochemical and biotechnological applications related to proteins. ${ }^{9,14}$ However, for salts, preferential-interaction coefficients cannot be entirely interpreted in terms of salt enrichment or reduction in the local domain. Since proteins are normally charged, a common-ion effect exists. ${ }^{10,12,15,16}$ This introduces a negative contribution to the experimentally determined preferential-interaction coefficients, ${ }^{12}$ which may be significant even at high salt concentrations. ${ }^{17,18}$ Hence, when preferential-interaction coefficients are examined, it is important to be able to quantitatively separate the common-ion contribution from salt-specific effects. Neglecting the common-ion effect may lead to inaccurate interpretation of protein-salt interactions. This is particularly critical for salts that are known to promote protein unfolding, for which negative values of preferential-interaction coefficients may be apparently inconsistent with the proteindestabilizing effect of the salt.

Protein-salt thermodynamic interactions are also central for understanding diffusive mass transport of proteins. Indeed, a gradient of salt concentration can generate a gradient of protein chemical potential responsible for protein diffusion and vice versa. This implies that the diffusion of salt and protein are coupled. ${ }^{10,19,20}$ In the case of a ternary protein-salt-water 
TABLE 1: Ternary Diffusion Coefficients for the Lysozyme- $-\mathrm{CaCl}_{2}-\mathrm{H}_{2} \mathrm{O}$ System at $25{ }^{\circ} \mathrm{C}$ and $\mathrm{pH} 4.5$

\begin{tabular}{lcc}
\hline $\bar{C}_{1}(\mathrm{mM})$ & 0.5999 & 0.6000 \\
$\bar{C}_{2}(\mathrm{M})$ & 0.0700 & 0.1500 \\
$\bar{V}_{0}\left(\mathrm{~cm}^{3} \mathrm{~mol}^{-1}\right)$ & 18.067 & 18.065 \\
$\underline{V}_{1}\left(\mathrm{~cm}^{3} \mathrm{~mol}^{-1}\right)$ & 10180 & 10220 \\
$V_{2}\left(\mathrm{~cm}^{3} \mathrm{~mol}^{-1}\right)$ & 20.68 & 21.60 \\
$\left(D_{11}\right)_{\mathrm{v}}\left(10^{-9} \mathrm{~m}^{2} \mathrm{~s}^{-1}\right)$ & $0.1316 \pm 0.0003$ & $0.1227 \pm 0.0001$ \\
$\left(D_{12}\right)_{\mathrm{v}}\left(10^{-12} \mathrm{~m}^{2} \mathrm{~s}^{-1}\right)$ & $0.986 \pm 0.009$ & $0.447 \pm 0.007$ \\
$\left(D_{21}\right)_{\mathrm{v}}\left(10^{-9} \mathrm{~m}^{2} \mathrm{~s}^{-1}\right)$ & $3.2 \pm 0.2$ & $3.9 \pm 0.1$ \\
$\left(D_{22}\right)_{\mathrm{v}}\left(10^{-9} \mathrm{~m}^{2} \mathrm{~s}^{-1}\right)$ & $1.121 \pm 0.002$ & $1.102 \pm 0.001$ \\
$\left(D_{11}\right)_{0}\left(10^{-9} \mathrm{~m}^{2} \mathrm{~s}^{-1}\right)$ & 0.1325 & 0.1235 \\
$\left(D_{12}\right)_{0}\left(10^{-12} \mathrm{~m}^{2} \mathrm{~s}^{-1}\right)$ & 1.006 & 0.464 \\
$\left(D_{21}\right)_{0}\left(10^{-9} \mathrm{~m}^{2} \mathrm{~s}^{-1}\right)$ & 3.3 & 4.1 \\
$\left(D_{22}\right)_{0}\left(10^{-9} \mathrm{~m}^{2} \mathrm{~s}^{-1}\right)$ & 1.123 & 1.106
\end{tabular}

solution, protein-salt-coupled diffusion is described by a matrix of four diffusion coefficients and is described by the extended Fick's first law:21,22

$$
\begin{aligned}
& -J_{1}=D_{11} \nabla C_{1}+D_{12} \nabla C_{2} \\
& -J_{2}=D_{21} \nabla C_{1}+D_{22} \nabla C_{2}
\end{aligned}
$$

Here, $C_{1}$ and $C_{2}$ are molar concentrations of the two solutes, $J_{1}$ and $J_{2}$ are the corresponding molar fluxes, and the four $D_{i j}$ 's (with $i, j=1,2$ ) are the diffusion coefficients. Main-diffusion coefficients, $D_{11}$ and $D_{22}$, describe the flux of a solute due to its own concentration gradient, whereas the cross-diffusion coefficients, $D_{12}$ and $D_{21}$, are responsible for the flux of a solute due to the concentration gradient of the other solute.

Clearly, the diffusion matrix can be used to probe proteinsalt interactions. We have, indeed, used ternary diffusion measurements for determining the effect of several salts $(\mathrm{NaCl}$, $\mathrm{KCl}, \mathrm{NH}_{4} \mathrm{Cl}$, and $\mathrm{MgCl}_{2}$ ) on the lysozyme chemical potential and to extract preferential-interaction coefficients. ${ }^{10,17,18,23}$ The examination of the four diffusion coefficients together with the extracted thermodynamic properties has allowed us to reveal the presence of a significant common-ion effect, even at high salt concentrations.

To elucidate the effect of destabilizing salts on both the transport and thermodynamic properties of protein aqueous solutions, we report here ternary diffusion measurements on the lysozyme- $\mathrm{CaCl}_{2}$-water system at $25^{\circ} \mathrm{C}$ and $\mathrm{pH} 4.5$. We have chosen $\mathrm{CaCl}_{2}$ because the calcium ion is known to preferentially promote protein unfolding. ${ }^{13,24}$ Experiments were performed at $0.6 \mathrm{mM}(\approx 8.6 \mathrm{mg} / \mathrm{mL})$ lysozyme, and $0.07-2.3 \mathrm{M} \mathrm{CaCl}_{2}$ concentrations.

Our experimental results for the lysozyme- $-\mathrm{CaCl}_{2}-$ water system are also compared with those previously obtained for the lysozyme- $\mathrm{MgCl}_{2}$-water system. ${ }^{18}$ Since both $\mathrm{Ca}^{2+}$ and $\mathrm{Mg}^{2+}$ ions are known to play crucial roles in cellular functions, the observed differences between the two investigated systems correlate with the very different roles that these two divalent cations play in the biological processes. ${ }^{25}$

\section{Materials and Methods}

Materials. Six-times recrystallized and lyophilized egg-white lysozyme (HEWL) was purchased from Seikagaku America and used without further purification. A protein-water stock solution was prepared by weight using double-distilled water. The molecular weight of HEWL was taken to be $14307 \mathrm{~g} \mathrm{~mol}^{-1}$. Corrections were made for the chloride ion weight fraction in the HEWL samples as shown in ref 19. Mallinckrodt $\mathrm{EM} \mathrm{CaCl} \mathrm{Cl}_{2}$. $2 \mathrm{H}_{2} \mathrm{O}$ was used without further purification. $\mathrm{A} \mathrm{CaCl}_{2}$-water stock solution was prepared, and its density was carefully measured at $25.00{ }^{\circ} \mathrm{C}$. This density value was used to calculate $\mathrm{CaCl}_{2}$ concentration from the available density-concentration relation. ${ }^{26}$

$\begin{array}{ccc}0.6003 & 0.6014 & 0.6016 \\ 0.5003 & 1.0020 & 2.3060 \\ 18.046 & 18.012 & 17.847 \\ 10240 & 10250 & 10330 \\ 24.52 & 26.88 & 32.16 \\ 0.1067 \pm 0.0001 & 0.0914 \pm 0.0002 & 0.0604 \pm 0.0002 \\ 0.176 \pm 0.002 & 0.149 \pm 0.006 & 0.160 \pm 0.006 \\ 7.5 \pm 0.1 & 13.7 \pm 0.1 & 31.5 \pm 0.2 \\ 1.136 \pm 0.001 & 1.205 \pm 0.002 & 1.295 \pm 0.002 \\ 0.1075 & 0.0922 & 0.0615 \\ 0.194 & 0.170 & 0.188 \\ 8.2 & 15.1 & 35.6 \\ 1.151 & 1.240 & 1.404\end{array}$

Methods. The Gosting diffusiometer and its modifications, density measurements, solution preparation, $\mathrm{pH}$ adjustment, and data reduction procedures are described in our previous papers. ${ }^{10,19}$ In brief, solutions for diffusion measurements were prepared by adding known weights of lysozyme and $\mathrm{CaCl}_{2}$ stock solutions. Water was then used to dilute the solution almost to the final volume. The $\mathrm{pH}$ of the solution was then adjusted to 4.5 by using few drops of a $\mathrm{HCl}-$ water stock solution ( $\mathrm{pH} \mathrm{1.2).}$ A Corning $135 \mathrm{pH}$ meter with an Orion 8102 ROSS combination $\mathrm{pH}$ electrode, standardized with Corning reference solutions, was used to measure the $\mathrm{pH}$. The final volume was then reached by adding water, and the $\mathrm{pH}$ was remeasured to confirm its value of 4.5 .

Ternary mutual diffusion coefficients were measured at $25.00{ }^{\circ} \mathrm{C}$ with the Gosting diffusiometer operating in the Rayleigh interferometric optical mode. The refractive-index profile inside a diffusion cell is measured as described in ref 27 and references therein. We obtained 50 refractive-index profiles during the course of each experiment. Experiments were performed by the free-diffusion method in a $10 \mathrm{~cm}$ vertical diffusion cell with a $2.5 \mathrm{~cm}$ horizontal optical path length and a $0.3 \mathrm{~cm}$ width. The temperature was regulated to $\pm 0.001{ }^{\circ} \mathrm{C}$ precision and $\pm 0.01{ }^{\circ} \mathrm{C}$ accuracy. Initial step-function distributions of solute concentrations were prepared with the boundary located at the center of the cell. All experimental data were obtained before detectable concentration changes occurred at the top and bottom ends of the cell, consistent with the freediffusion boundary condition. A minimum of two experiments is required for determining the four diffusion coefficients at a given set of mean concentrations $\left(\overline{\mathrm{C}}_{1}, \overline{\mathrm{C}}_{2}\right.$ in Table 1$)$. These two experiments must have different combinations of solute concentration differences across the diffusion boundary. To verify reproducibility, two other duplicate experiments were performed at each set of mean concentrations.

\section{Results}

We report diffusion coefficients relative to volume-fixed (subscript V) and solvent-fixed (subscript 0) frames. ${ }^{28}$ Diffusion coefficients are measured in the laboratory-fixed frame, which is an excellent approximation of the $\left(D_{i j}\right)_{\mathrm{V}}$ values in the volumefixed frame within our experimental conditions. The $\left(D_{i j}\right)_{0}$ values are easily calculated (eq 2 of ref 29) from the $\left(D_{i j}\right)_{\mathrm{V}}$ values and the partial molar volumes, $\overline{\mathrm{V}}_{i}$, obtained from density measurements. In Table 1 , we report the volume-fixed $\left(D_{i j}\right)_{\mathrm{V}}$ values calculated with data from all four experiments at each of the five $\mathrm{CaCl}_{2}$ concentrations. Table 1 also includes the density, $d$, and the partial molar volumes, $\bar{V}_{1}, \bar{V}_{2}, \bar{V}_{0}$, obtained from density measurements. Tables with detailed results for each diffusion experiment are available as Supporting Information.

In Figure $1 \mathrm{a}-\mathrm{d}$, we show the four diffusion coefficients as functions of $C_{2}$ at $C_{1}=0.6 \mathrm{mM}$ for the $\mathrm{CaCl}_{2}$ case. For comparison, we include the previously reported $\left(D_{i j}\right)_{\mathrm{V}}$ values 

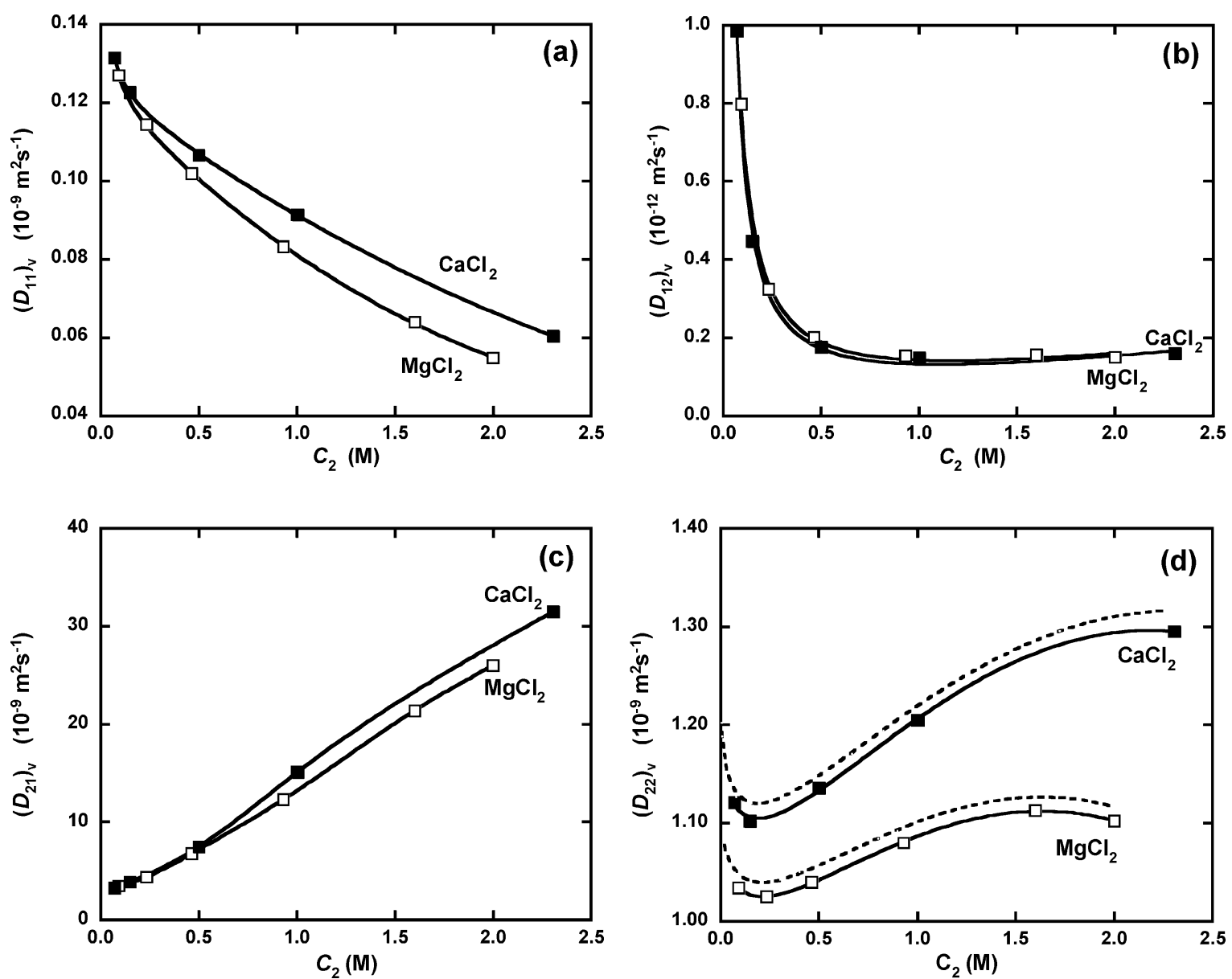

Figure 1. Volume-fixed diffusion coefficients as functions of salt concentration, $C_{2}$, for the lysozyme $-\mathrm{CaCl}_{2}-\mathrm{H}_{2} \mathrm{O}(\boldsymbol{\square})$ and the lysozyme$\mathrm{MgCl}_{2}-\mathrm{H}_{2} \mathrm{O}$ (口) systems at $C_{1}=0.6 \mathrm{mM}, \mathrm{pH} 4.5$, and $25^{\circ} \mathrm{C}$ : (a) $\left(D_{11}\right)_{\mathrm{V}}$; (b) $\left(D_{12}\right)_{\mathrm{V}}$; (c) $\left(D_{21}\right)_{\mathrm{V}}$; (d) $\left(D_{22}\right)_{\mathrm{V}}$. The solid curves are smoothed through the ternary experimental points. The dashed curves are smoothed through the omitted binary diffusion coefficients for the salt components; data taken from ref $26\left(\mathrm{CaCl}_{2}\right)$ and ref $30\left(\mathrm{MgCl}_{2}\right)$.

for the $\mathrm{MgCl}_{2}$ case. In Figure 1d, we include binary salt-water data (dashed curves), ${ }^{26,30}$ together with the $\left(D_{22}\right)_{\mathrm{V}}$ ternary results. The ternary values are just $1-2 \%$ lower than the corresponding binary values. This small difference can be related to the protein obstruction effect on the salt-ion mobility. ${ }^{10}$ We shall examine the behavior of the other three diffusion coefficients in the following section.

From irreversible thermodynamics, the fundamental driving forces for diffusion are the chemical potential gradients of the mixture components. Thus, ternary diffusion coefficients can be described in terms of the Onsager diffusion coefficients (ODCs) $L_{i j}$ and the chemical potential derivatives, $\mu_{i j} \equiv$ $\left(\partial \mu_{i} / \partial C_{j}\right)_{T, p, C_{k}, k \neq j}$, where $T$ is the temperature and $p$ is the pressure. For solvent-fixed $\left(D_{i j}\right)_{0}$, we can write

$$
\begin{aligned}
& \left(D_{11}\right)_{0}=\left(L_{11}\right)_{0} \mu_{11}+\left(L_{12}\right)_{0} \mu_{21} \\
& \left(D_{12}\right)_{0}=\left(L_{11}\right)_{0} \mu_{12}+\left(L_{12}\right)_{0} \mu_{22} \\
& \left(D_{21}\right)_{0}=\left(L_{21}\right)_{0} \mu_{11}+\left(L_{22}\right)_{0} \mu_{21} \\
& \left(D_{22}\right)_{0}=\left(L_{21}\right)_{0} u_{12}+\left(L_{22}\right)_{0} \mu_{22}
\end{aligned}
$$

and $\left(L_{12}\right)_{0}=\left(L_{21}\right)_{0}$ represents the Onsager reciprocal relation. ${ }^{31-34}$ Equations $2 \mathrm{a}-\mathrm{d}$ can be solved with respect to $\mu_{12}$ and $\mu_{21}$. In Table 2 , we report the values of $\mu_{11} / R T, \mu_{22} / R T, \mu_{12} / R T$, and $\mu_{21} / R T$ obtained using the procedures described in refs 10 and 17. The values of $\mu_{22} / R T$ were calculated using activitycoefficient data of the binary salt-water system. ${ }^{26}$ Table 2 also includes the calculated values of the Onsager diffusion coefficients, $\left(L_{i j}\right)_{0}$, obtained using eq 7 of ref 10 .

In Figure 2a, b, we report the calculated cross-chemical potential derivatives $\mu_{12} / R T$ and $\mu_{21} / R T$ for the $\mathrm{CaCl}_{2}$ case as a function of salt concentration. In the same figure, we include our results for the $\mathrm{MgCl}_{2}$ case. At low salt concentrations, the values of $\mu_{12} / R T$ and $\mu_{21} / R T$ sharply decrease. This is consistent with the presence of the common-ion effect that varies approximately as $z_{\mathrm{p}} / C_{2} \cdot{ }^{17}$ In the following section, we will use these cross-chemical-potential derivatives to calculate preferentialinteraction coefficients.

\section{Discussion}

In this section, we will first examine the diffusion properties of the lysozyme- $\mathrm{CaCl}_{2}$-water system and compare them with those previously characterized for the lysozyme $-\mathrm{MgCl}_{2}-$ water system at the same $\mathrm{pH}$ and temperature. Examination of the diffusion coefficients will allow us to probe the presence of common-ion effects and the differences in transport behavior between these two divalent salts. We will then examine proteinsalt thermodynamic interactions using the extracted preferentialinteraction coefficients.

Ternary diffusion of charged macromolecules in the presence of salts can be discussed in terms of the four Nernst-Hartley $(\mathrm{N}-\mathrm{H})$ equations. $^{21}$ According to these equations, the four diffusion coefficients of two ionic components with a common ion are related to the ionic mobilities of the three constituent ionic species. Due to electrostatic coupling (i.e., electroneutral- 
TABLE 2: Chemical Potential Derivatives and Onsager Diffusion Coefficients for the Lysozyme $-\mathrm{CaCl}_{2}-\mathrm{H}_{2} \mathrm{O}$ System

\begin{tabular}{cccccccc}
\hline$C_{2}(\mathrm{M})$ & $\mu_{11} / R T\left(\mathrm{M}^{-1}\right)$ & $\mu_{22} / R T\left(\mathrm{M}^{-1}\right)$ & $\mu_{12} / R T\left(\mathrm{M}^{-1}\right)$ & $\mu_{21} / R T\left(\mathrm{M}^{-1}\right)$ & $R T\left(L_{11}\right)_{0} / C_{1}\left(10^{-9} \mathrm{~m}^{2} \mathrm{~s}^{-1}\right)$ & $R T\left(L_{12}\right)_{0} / C_{1}\left(10^{-9} \mathrm{~m}^{2} \mathrm{~s}^{-1}\right)$ & $R T\left(L_{22}\right)_{0} / C_{2}$ \\
\hline 0.070 & 2007 & 35.414 & 86.7 & 112.5 & 0.131 & -0.27 \\
0.150 & 1828 & 17.223 & 41.0 & 67.7 & 0.125 & -0.25 & 0.459 \\
0.500 & 1715 & 6.232 & 13.0 & 45.1 & 0.110 & -0.18 \\
1.002 & 1687 & 4.149 & 8.0 & 50.7 & 0.095 & -0.11 \\
2.306 & 1673 & 3.537 & 5.3 & 89.7 & 0.061 & 0.370 \\
& & & & & & 0.00
\end{tabular}

ity), the three ionic species cannot move independently. Although the $\mathrm{N}-\mathrm{H}$ equations are only exact in the limits of both $C_{1}$ and $C_{2}$ approaching zero, they still provide an important reference model for interpreting our experimental results. Due to their explicit dependence on ionic mobilities and protein charge, they are very valuable for assessing the presence of significant common-ion effects.

One important feature of our protein-salt systems is that the molar concentration of lysozyme is significantly lower than the salt concentration; that is, $z_{\mathrm{p}} C_{1} \ll 2 C_{2}$. This condition will be used throughout our discussion. As shown in ref 18 , the $\mathrm{N}-\mathrm{H}$ equations for lysozyme in the presence of divalent metal ions are

$$
\begin{aligned}
& D_{11}=\tilde{D}_{\mathrm{p}}\left(1+\frac{z_{\mathrm{p}}^{2} C_{1}}{2 C_{2}} \frac{\tilde{D}_{\mathrm{Cl}}-\tilde{D}_{\mathrm{p}}}{2 \tilde{D}_{\mathrm{M}}+\tilde{D}_{\mathrm{Cl}}}\right) \\
& D_{12}=\tilde{D}_{\mathrm{p}} \frac{z_{\mathrm{p}} C_{1}}{C_{2}} \frac{\tilde{D}_{\mathrm{Cl}}-\tilde{D}_{\mathrm{M}}}{2 \tilde{D}_{\mathrm{M}}+\tilde{D}_{\mathrm{Cl}}} \\
& D_{21}=z_{\mathrm{p}} \frac{\tilde{D}_{\mathrm{M}}\left(\tilde{D}_{\mathrm{Cl}}-\tilde{D}_{\mathrm{p}}\right)}{2 \tilde{D}_{\mathrm{M}}+\tilde{D}_{\mathrm{Cl}}} \\
& D_{22}=\frac{3 \tilde{D}_{\mathrm{M}} \tilde{D}_{\mathrm{Cl}}}{2 \tilde{D}_{\mathrm{M}}+\tilde{D}_{\mathrm{Cl}}}
\end{aligned}
$$

where $\tilde{D}_{\mathrm{p}}, \tilde{D}_{\mathrm{M}}$, and $\tilde{D}_{\mathrm{Cl}}$ are, respectively, the tracer diffusion coefficients of the protein cation, salt co-ion $\left(\mathrm{Ca}^{2+}\right.$ or $\left.\mathrm{Mg}^{2+}\right)$, and common ion $\left(\mathrm{Cl}^{-}\right)$. The value of $\tilde{D}_{p}=0.132 \times 10^{-9} \mathrm{~m}^{2}$ $\mathrm{s}^{-1}$ for lysozyme (in aqueous $\mathrm{NaCl}$ at $\mathrm{pH} 4.5$ and $25^{\circ} \mathrm{C}$ ), was taken from ref 27 . The tracer diffusion coefficients for the small ions have been calculated from limiting ionic mobilities at $25{ }^{\circ} \mathrm{C}$. For $\mathrm{Ca}^{2+}, \mathrm{Mg}^{2+}$, and $\mathrm{Cl}^{-}$, they are $0.792,0.706$, and $2.03 \times 10^{-9} \mathrm{~m}^{2} \mathrm{~s}^{-1}$ respectively. ${ }^{35}$

Equations $3 \mathrm{a}-\mathrm{d}$ explicitly show the dependence of the four diffusion coefficients on the salt concentration. According to these equations, we can predict that (1) the coefficients $D_{11}$ and $D_{12}$ are expected to sharply decrease as $C_{2}$ increases due to the

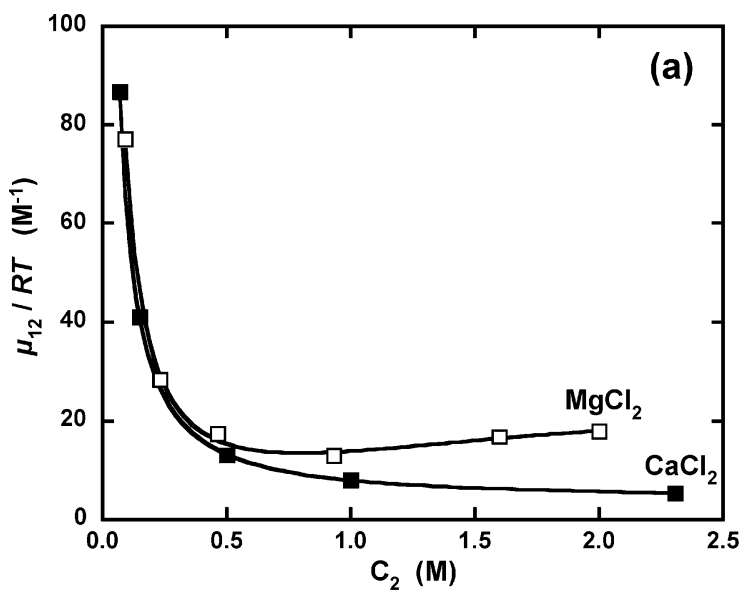

protein charge; (2) the ratio $D_{21} / D_{22}$ is approximately equal to $z_{\mathrm{p}} / 3$, since $\tilde{\mathrm{D}}_{p} \ll \tilde{\mathrm{D}}_{\mathrm{Cl}}$. Our experimental results at the lower salt concentrations are, indeed, consistent with these predictions.

We will now examine our ternary diffusion coefficients. We will address the behavior of the solvent-frame $\left(D_{i j}\right)_{0}$ 's in particular, due to their more direct connection to our thermodynamic results. Nevertheless, the difference between the values of $\left(D_{i j}\right)_{\mathrm{V}}$ and $\left(D_{i j}\right)_{0}$ is small and irrelevant for the interpretations reported below.

Examination of $\mathbf{D}_{11}$. Values of $\left(D_{11}\right)_{\mathrm{V}}$ for the lysozyme$\mathrm{CaCl}_{2}$ and lysozyme $-\mathrm{MgCl}_{2}$ systems are shown in Figure 1a. For both salt cases, the diffusion coefficient $D_{11}$ (i.e., $\left(D_{11}\right)_{\mathrm{V}}$ and $\left.\left(D_{11}\right)_{0}\right)$ significantly decreases as the salt concentration increases. At $C_{2} \approx 2 \mathrm{M}, D_{11}$ reduces to $\approx 50 \%$ of its value at the lowest salt concentrations. We note that the decrease in $D_{11}$ for the $\mathrm{CaCl}_{2}$ case is somewhat smaller than that for the $\mathrm{MgCl}_{2}$ case. Part of the observed dependence of $D_{11}$ on salt concentration can be related to solution viscosity. According to the Stokes-Einstein equation, ${ }^{15}$ we expect the $D_{11}$ decreases as the solution viscosity increases. To examine the effect of viscosity, we consider the product $\left(D_{11}\right)_{0}\left(\eta / \eta_{0}\right)$, where $\eta$ is the viscosity of the corresponding binary salt-water systems ${ }^{36}$ and $\eta_{0}$ is the viscosity of water. In Figure 3, we plot $\left(D_{11}\right)_{0}\left(\eta / \eta_{0}\right)$ as a function of $C_{2}$. Clearly, the viscosity correction removes much of the $D_{11}$ dependence on salt type. Indeed, at low salt concentrations, the two curves virtually overlap, and remarkably, their difference is always $<2 \%$ within the whole experimental concentration domain.

At low salt concentration, the observed sharp decrease of $\left(D_{11}\right)_{0}\left(\eta / \eta_{0}\right)$ is consistent with eq 3 a. Furthermore, using $\mathrm{N}-\mathrm{H}$ equations, we predict that the $\approx 10 \%$ difference in ionic mobility between the two metal ions have a negligible effect $(\approx 0.1 \%)$ on the difference between the two corresponding $D_{11}$ curves. This is consistent with our experimental findings.

Interestingly, both curves in Figure 3 display a minimum at $C_{2} \approx 1 \mathrm{M}$. If conformational-change effects on $\tilde{D}_{\mathrm{p}}$ are not significant, our results at high salt concentrations can be related to an increase in $\mu_{11}$ (see eq $2 \mathrm{a}$ ) due to thermodynamic nonideality. This result is consistent with the general observation

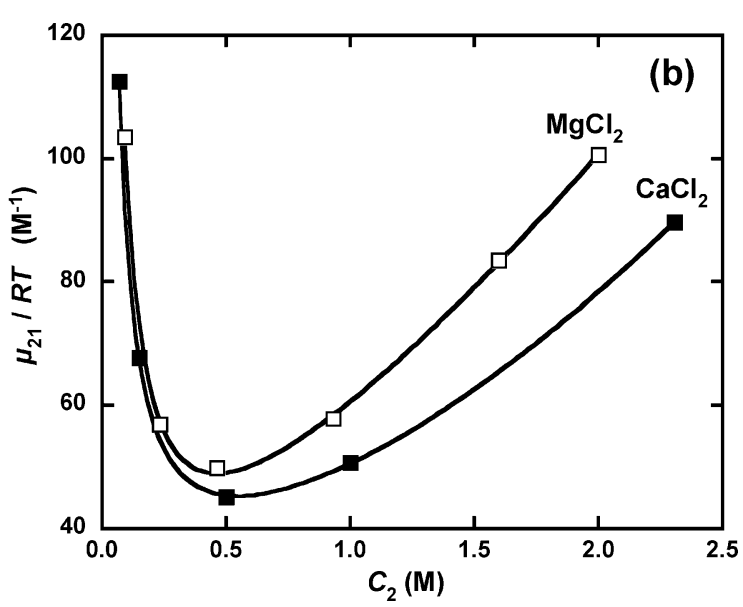

Figure 2. Chemical potential cross-derivatives as a function of salt concentration, $C_{2}$, for the lysozyme $-\mathrm{CaCl}_{2}-\mathrm{H}_{2} \mathrm{O}(\mathbf{\square})$ and the lysozyme$\mathrm{MgCl}_{2}-\mathrm{H}_{2} \mathrm{O}(\square)$ systems. The solid curves are fits to the data described in refs 10, 17, 18. 


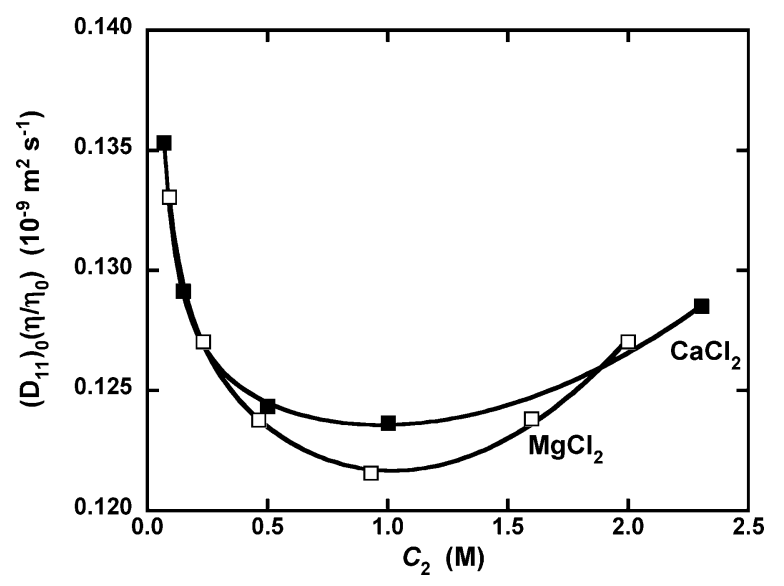

Figure 3. Viscosity-corrected $\left(D_{11}\right)_{0}\left(\eta / \eta_{0}\right)$ as a function of salt concentration, $C_{2}$, for the lysozyme $-\mathrm{CaCl}_{2}-\mathrm{H}_{2} \mathrm{O}(\boldsymbol{\square})$ and the lysozyme$\mathrm{MgCl}_{2}-\mathrm{H}_{2} \mathrm{O}(\square)$ systems. The solid curves are smoothed through the ternary experimental points. The viscosity coefficients of the binary salt-water systems were taken from ref 36 .

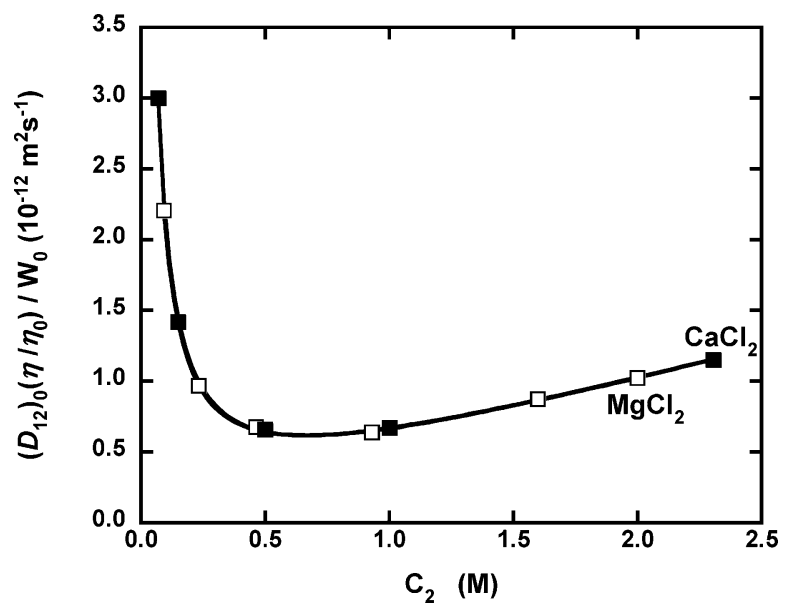

Figure 4. Corrected cross-diffusion coefficient $\left(D_{12}\right)_{0}\left(\eta / \eta_{0}\right) / W_{0}$ as a function of salt concentration, $C_{2}$, for the lysozyme- $-\mathrm{CaCl}_{2}-\mathrm{H}_{2} \mathrm{O}(\mathbf{\square})$ and the lysozyme- $\mathrm{MgCl}_{2}-\mathrm{H}_{2} \mathrm{O}(\square)$ systems. The solid curves are smoothed through the ternary experimental points.

that divalent metal ions induce protein-protein repulsive interactions at high salt concentrations. ${ }^{37}$

Examination of $\mathbf{D}_{\mathbf{1 2}}$. Values of $\left(D_{12}\right)_{\mathrm{V}}$ for the lysozyme$\mathrm{CaCl}_{2}$ and lysozyme- $\mathrm{MgCl}_{2}$ systems are shown in Figure $1 \mathrm{~b}$. This cross-diffusion coefficient describes how the flux of lysozyme is affected by the salt concentration gradient. In Figure $1 \mathrm{~b}$, we can see that $D_{12}$ sharply decreases as $C_{2}$ increases for both salt cases. This behavior is qualitatively predicted by eq $3 b$.

On the basis of eq $3 \mathrm{~b}$ and the effect of the viscosity on $\tilde{D}_{\mathrm{p}}$, a quantitative comparison between the two salt cases can be performed by introducing the normalized diffusion coefficient $\left(D_{12}\right)_{0}\left(\eta / \eta_{0}\right) / W_{0}$, where $W_{0}=\left(\tilde{D}_{\mathrm{Cl}}-\tilde{D}_{\mathrm{M}}\right) /\left(2 \tilde{D}_{\mathrm{M}}+\tilde{D}_{\mathrm{Cl}}\right)$ is the mobility ratio of eq $3 b{ }^{17,18}$ Indeed, $W_{0}$ and the viscosity correction, $\left(\eta / \eta_{0}\right)$, are the only two properties that explicitly depend on the type of salt. In Figure 4, we can see that there is virtually no detectable difference in $\left(D_{12}\right)_{0}\left(\eta / \eta_{0}\right) / W_{0}$ between the two salt cases at any given concentration within the experimental range. The observed increase in $\left(D_{12}\right)_{0}\left(\eta / \eta_{0}\right) / W_{0}$ at high salt concentrations can be attributed to the large thermodynamic nonideality of the two salt-water binary systems, ${ }^{26,30}$ which affects in the same way both $\mu_{12}$ and $\mu_{22}$ in eq 2 b. In other words, the ratio of salt activity to salt concentration gradient significantly increases with salt concen-
TABLE 3: Thermodynamic Parameters for the Lysozyme $-\mathrm{CaCl}_{2}-\mathrm{H}_{2} \mathrm{O}$ System

\begin{tabular}{cccccc}
\hline$C_{2}(\mathrm{M})$ & $\left(D_{21}\right)_{0} /\left(D_{22}\right)_{0}$ & $\mu_{21} / \mu_{22}$ & $-\Gamma_{\mu_{2}}$ & $\begin{array}{c}\mu_{12}^{(m)} / R T \\
\left(\mathrm{~kg} \mathrm{~mol}^{-1}\right)\end{array}$ & $\begin{array}{c}\mu_{22}^{(m)} / R T \\
\left(\mathrm{~kg} \mathrm{~mol}^{-1}\right)\end{array}$ \\
\hline 0.070 & 2.94 & 3.18 & 2.45 & 85.65 & 34.993 \\
0.150 & 3.71 & 3.93 & 2.38 & 40.35 & 16.959 \\
0.500 & 7.12 & 7.24 & 2.08 & 12.56 & 6.031 \\
1.002 & 12.18 & 12.22 & 1.92 & 7.50 & 3.904 \\
2.306 & 25.36 & 25.36 & 1.49 & 4.53 & 3.038
\end{tabular}

tration. Hence, the corresponding thermodynamic driving force responsible for the protein flux will also increase.

Examination of $\mathbf{D}_{21}$. Values of $\left(D_{21}\right)_{\mathrm{V}}$ for the lysozyme$\mathrm{CaCl}_{2}$ and lysozyme- $\mathrm{MgCl}_{2}$ systems are shown in Figure $1 \mathrm{c}$. The $D_{21}$ values increase with salt concentration in both cases. Since the salt component is present in large excess, $D_{21}$ should not depend on $C_{2}$, according to eq $3 \mathrm{c}$. However, contrary to the $\mathrm{N}-\mathrm{H}$ prediction, $D_{21}$ significantly increases with $C_{2}$. This salt-concentration dependence can be explained by excludedvolume effects of the protein molecules on the salt ions; that is, a gradient of protein concentration in a solution in which the salt component has a uniform stoichiometric concentration $C_{2}$ will produce a gradient of interstitial salt concentration. ${ }^{18}$

We further observe that the values of $D_{21}$ for $\mathrm{CaCl}_{2}$ are higher than those obtained for $\mathrm{MgCl}_{2}$. This is expected, since $D_{21}$ is proportional to $D_{22}$ according to eqs $3 \mathrm{a}$, d. Thus, a better comparison between the two salt cases is provided by the examination of the ratio $D_{21} / D_{22}$. We have previously shown that (consistent with our data in Table $3 a, b$ ) this ratio can be used to directly probe the thermodynamic behavior of the system. This is because ${ }^{17,18}$

$$
\frac{\left(D_{21}\right)_{0}}{\left(D_{22}\right)_{0}} \approx \frac{\mu_{21}}{\mu_{22}}
$$

This important result is related to the relatively low mobility of proteins. That is, we can approximately describe our system as one in which the lysozyme molecules occupy fixed positions and the fast salt ions partition between high and low protein concentration. When the salt component (and the solvent) reaches equilibrium ( $\mu_{2}$ constant) within this "fixed" gradient of protein, we reach $\left(J_{2}\right)_{0}=0$. Then, according to eq $1 \mathrm{~b}$, this gives $\left(D_{21}\right)_{0} /\left(D_{22}\right)_{0}=-\left(\partial C_{2} / \partial C_{1}\right)_{\mu_{2}}=\mu_{21} / \mu_{22}$, consistent with our findings. This argument shows the direct relation of ternary diffusion to equilibrium dialysis. In the latter case, the salt partitions ( $\mu_{2}$ constant) between two domains of different protein concentrations separated by a membrane that does not allow the protein to diffuse through. It is important to remark that we use rigorous equations to determine $\mu_{12}$ and $\mu_{21}$ (eqs $12 \mathrm{a}$, b of ref 10$)$. Yet, the above description provides the rationale on why ternary diffusion is valuable in determining protein-salt thermodynamic interactions.

In Figure 5, we report the $\left(D_{21}\right)_{0} /\left(D_{22}\right)_{0}$ values for both salt cases as a function of $C_{2}$. The corresponding curves are approximately linear. We have previously shown that this behavior can be rationalized by using the following relation, ${ }^{18}$

$$
\frac{\left(D_{21}\right)_{0}}{\left(D_{22}\right)_{0}} \approx \frac{z_{\mathrm{P}}}{3}+C_{2} V_{\mathrm{ex}}
$$

where the intercept, $z_{\mathrm{p}} / 3$, is related to the polyelectrolyte nature of the protein and, consequently, the common-ion effect. The slope, $V_{\mathrm{ex}}$, is the excluded volume due to presence of the protein macromolecules. This value is comparable to the protein partial molar volume, $\bar{V}_{1}$. For both salt cases, we obtain the same 


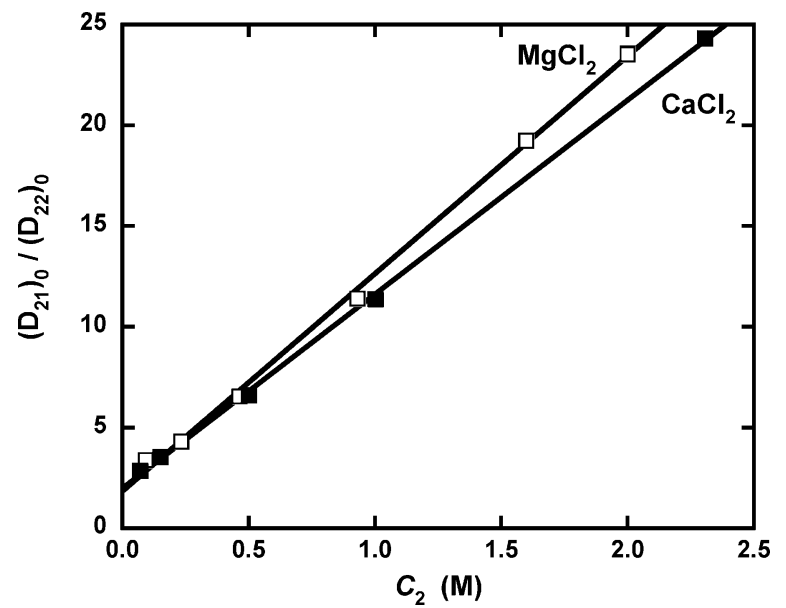

Figure 5. The quotient $\left(D_{21}\right)_{0} /\left(D_{22}\right)_{0}$ as a function of salt concentration, $C_{2}$, for the lysozyme- $-\mathrm{CaCl}_{2}-\mathrm{H}_{2} \mathrm{O}(\mathbf{\square})$ and the lysozyme $-\mathrm{MgCl}_{2}-$ $\mathrm{H}_{2} \mathrm{O}(\square)$ systems. Straight lines are fits through the data.

intercept value within the experimental error (see Figure 5). On the other hand, the slope for the $\mathrm{CaCl}_{2}$ case is $\approx 10 \%$ smaller than that for the $\mathrm{MgCl}_{2}$ case. This represents the most important difference in diffusion properties between the two ternary systems. Due to the thermodynamic nature of the ratio $\left(D_{21}\right)_{0} /$ $\left(D_{22}\right)_{0}$, we will discuss this difference in terms of preferentialinteraction coefficients.

Preferential-Interaction Coefficients. Protein-salt thermodynamic interactions are conveniently described by the preferential-interaction coefficient $\Gamma_{\mu_{2}}, 11,22$

$$
\Gamma_{\mu_{2}}=\lim _{m_{1} \rightarrow 0}\left(\frac{\partial m_{2}}{\partial m_{1}}\right)_{T, \mu_{2}}=-\lim _{m_{1} \rightarrow 0} \frac{\mu_{12}^{(m)}}{\mu_{22}^{(m)}}
$$

where $\mu_{12}^{(m)} \equiv\left(\partial \mu_{1} / \partial m_{2}\right)_{T, p, m_{1}}=\left(\partial \mu_{2} / \partial m_{1}\right)_{T, p, m_{2}}$ and $\mu_{22}^{(m)} \equiv\left(\partial \mu_{2} /\right.$ $\left.\partial m_{2}\right)_{T, p, m_{1}}$ are the chemical potential derivatives of the salt chemical potential with respect to the molalities $m_{1}$ and $m_{2}$. The expression for the salt chemical potential used here is

$\left(\mu_{2}-\mu_{2}{ }^{0(m)}\right) / R T=\ln m_{2}+2 \ln \left(2 m_{2}+z_{\mathrm{P}} m_{1}\right)+3 \ln \gamma_{2}$

where $\mu_{2}{ }^{0(m)}$ and $\gamma_{2}$ are, respectively, the salt standard chemical potential and the mean activity coefficient based on molality. The values of $\mu_{12}^{(m)}$ and $\mu_{22}^{(m)}$ for the lysozyme- $\mathrm{CaCl}_{2}$ system, which were obtained using eqs $16 \mathrm{a}, \mathrm{b}$ of ref 18 , are reported in Table 3 together with $\Gamma_{\mu_{2}}$ calculated using eq 6 . It is important to remark that all values of $\Gamma_{\mu_{2}}$ are negative. Figure 6 shows the dependence of $\Gamma_{\mu_{2}}$ on salt concentration for the $\mathrm{CaCl}_{2}$ case together with that previously obtained for the lysozyme- $-\mathrm{MgCl}_{2}$ system. We can see that the two curves are approximately linear. For both salt cases, we obtain the same intercept within the experimental error. However, the slope is negative for the $\mathrm{CaCl}_{2}$ case, whereas it becomes positive for the $\mathrm{MgCl}_{2}$ case.

To gain insight into the observed behavior, we report an expression for $\Gamma_{\mu_{2}}$ from differentiation of eq 7 at constant $\mu_{2}$. In the limit of small $m_{1}$, we obtain

$$
\Gamma_{\mu_{2}}=-\frac{z_{\mathrm{p}}}{3}-\chi_{21} m_{2}
$$

where $\chi_{21}=\left(\partial \ln \gamma_{2} / \partial m_{1}\right)_{\mu_{2}}$. Similar equations have been previously reported using a membrane equilibrium approach. ${ }^{12}$ The first term of eq 8, which represents the common-ion contribution, is negative. We remark that this term is a general characteristic of ternary electrolyte systems with a common ion.

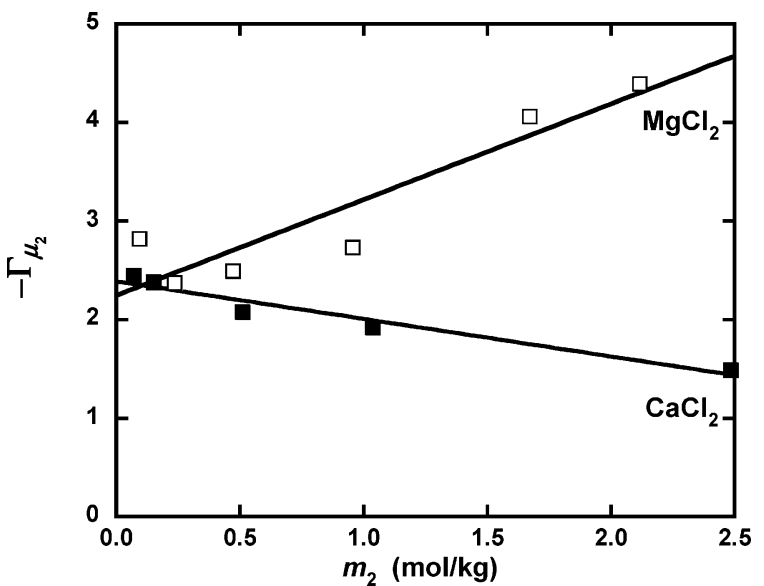

Figure 6. Preferential-interaction coefficients reported as $-\Gamma_{\mu_{2}}$ as a function of salt molality, $m_{2}$, for the lysozyme- $-\mathrm{CaCl}_{2}-\mathrm{H}_{2} \mathrm{O}$ (足) and the lysozyme $-\mathrm{MgCl}_{2}-\mathrm{H}_{2} \mathrm{O}(\square)$ systems.

For ternary systems in which at least one solute is a nonelectrolyte (e.g., the osmolyte is glycerol, urea, or a zwitterion), ${ }^{7}$ eq 8 reduces to $\Gamma_{\mu_{2}}=-\chi_{21} m_{2}$ and $\Gamma_{\mu_{2}}$ approaches zero when $m_{2} \rightarrow 0$.

Equation 8 is consistent with our experimental intercept values. For both salt cases, we find $z_{p}=7 \pm 1$ by linear regression. This $z_{\mathrm{p}}$ value is lower than the value $z_{\mathrm{p}}=11$ obtained by titration. ${ }^{38}$ This is expected, since the latter is based only on the degree of protonation and does not account for counterion binding.

The second term in eq 7 represents the nonideality contribution due to protein-salt specific interactions. According to our results, $\chi_{21}$ is approximately constant. This is consistent with the two-domain model. ${ }^{2,12}$ As described in the introduction, this model assumes the local domain of the protein molecules to be in chemical equilibrium with a bulk unperturbed domain. Record and Anderson ${ }^{12}$ applied this model to macromolecules in the presence of 1:1 electrolytes. A generalization to electrolytes with polyvalent cations is reported in the Appendix. Here, we introduce the partitioning constant

$$
\alpha=\frac{\left(N_{2} / N_{0}\right)}{\left(m_{2} / m_{0}\right)}
$$

where $m_{0}=55.51 \mathrm{~mol} \mathrm{~kg}-1, N_{2} \equiv\left(N_{\mathrm{C}}+N_{\mathrm{M}}\right) / 3$, and $N_{\mathrm{C}}, N_{\mathrm{M}}$, and $N_{0}$ are, respectively, the number of chloride ions, divalent metal ions, and water molecules of one protein layer. When $\alpha$ $<1$, salt is preferentially excluded from the protein domains. On the other hand, when $\alpha>1$, salt preferentially interacts with the protein molecules. Within the two-domain model, $\chi_{21}$ can be related to $\alpha$ by (see Appendix)

$$
\chi_{21}=\frac{N_{0}}{m_{0}}(1-\alpha)
$$

Since water is the component present in large excess, $N_{0}$ will not significantly change with salt concentration. This implies that $\chi_{21}$ is approximately constant. Applying linear regression to our experimental $\Gamma_{\mu_{2}}$ values, we obtain $\chi_{21}=-0.38 \pm$ $0.05 \mathrm{~mol}^{-1} \mathrm{~kg}$ for the $\mathrm{CaCl}_{2}$ case and $\chi_{21}=-1.0 \pm 0.2 \mathrm{~mol}^{-1}$ $\mathrm{kg}$ for the $\mathrm{MgCl}_{2}$ case. We therefore conclude that proteinsalt preferential interaction occurs for the $\mathrm{CaCl}_{2}$ case and not for the $\mathrm{MgCl}_{2}$ case. This is consistent with the known destabilizing effect of calcium ions on proteins. 
We can now explain the observed difference in $\left(D_{21}\right)_{0} /\left(D_{22}\right)_{0}$ between the two salt cases.

By examining the relation between the molality-based and the molarity-based chemical potential derivatives, we have found that $\mu_{21} / \mu_{22} \approx-\Gamma_{\mu_{2}}+C_{2} \bar{V}_{1} .{ }^{18}$ This result, together with eqs 4 and 8 and the approximation $m_{2} \approx C_{2}$, yields

$$
\frac{\left(D_{21}\right)_{0}}{\left(D_{22}\right)_{0}} \approx \frac{z_{\mathrm{P}}}{3}+\left(\bar{V}_{1}+\chi_{21}\right) C_{2}
$$

Hence, the difference in slope between the two $\left(D_{21}\right)_{0} /\left(D_{22}\right)_{0}$ curves in Figure 5 is directly related to the corresponding difference in $\chi_{21}$ between the two salt cases.

We finally remark that neglecting common-ion effects can be highly inaccurate for protein-salt aqueous systems. This is especially true for the lysozyme- $\mathrm{CaCl}_{2}$ system (away from the isoelectric point), where the approximation $\chi_{21} \approx-\Gamma_{\mu_{2}} / m_{2}$ would be clearly misleading. Furthermore, our results show that the common-ion contribution in $\Gamma_{\mu_{2}}$ is larger than $50 \%$, even at salt concentrations as high as $\approx 2 \mathrm{M}$. Hence, the examination of the $\Gamma_{\mu_{2}}$ dependence on salt concentration is crucial for assessing the nature of protein-salt specific interactions.

The difference between $\mathrm{CaCl}_{2}$ and $\mathrm{MgCl}_{2}$ in their net interaction with lysozyme is consistent with the generally larger affinity of $\mathrm{Mg}^{2+}$ for water compared to $\mathrm{Ca}^{2+}$. Indeed, the magnesium ion has a larger and tighter hydration layer due its higher charge density. This also correlates with their generally very different roles in biochemical processes. Calcium ions are usually involved in protein binding to modulate conformational changes. On the other hand, magnesium ions are usually involved in binding negatively charged phosphate groups and in holding water molecules close to the catalytic site of enzymes. $^{25}$

\section{Summary and Conclusions}

We have reported ternary diffusion coefficients for the lysozyme $-\mathrm{CaCl}_{2}-$ water system. A comparison with previous results on the lysozyme- $\mathrm{MgCl}_{2}-$ water ternary system has also been included. At low salt concentrations, the protein maindiffusion coefficient, $D_{11}$, and cross-diffusion coefficient, $D_{12}$, show the presence of a large protein-salt common-ion effect. At high salt concentrations, strong positive deviations from solution ideality are observed for these two coefficients. The salt diffusion coefficient ratio $D_{21} / D_{22}$ is essentially a thermodynamic property describing protein-salt interactions. This ratio, which linearly increases with salt concentration, can be separated into two parts. The first part, which is independent of salt concentration, can be related to the common-ion effect. The second part of this ratio, which can be related to proteinsalt specific interactions and excluded-volume interactions, is directly proportional to salt concentration. We have found that the differences in $D_{11}$ and $D_{12}$ between the $\mathrm{CaCl}_{2}$ and the $\mathrm{MgCl}_{2}$ cases can be explained by taking into account the difference in mobility between the two metal ions and viscosity of binary salt-water systems. On the other hand, the difference in $D_{21} /$ $D_{22}$ between the two salt cases is directly related to the corresponding difference in protein-salt preferential coefficients, $\Gamma_{\mu_{2}}$. By examining the dependence of $\Gamma_{\mu_{2}}$ on salt concentration, we have found that (1) the common-ion effect is essentially the same for both salt cases; and (2) salt preferentially interacts with the protein in the lysozyme- $\mathrm{CaCl}_{2}-$ water system, whereas water preferentially interacts with the protein in lysozyme- $\mathrm{MgCl}_{2}$-water system. The difference between $\mathrm{CaCl}_{2}$ and $\mathrm{MgCl}_{2}$ in their net interaction with lysozyme is consistent with the generally larger affinity of $\mathrm{Mg}^{2+}$ for water as compared to $\mathrm{Ca}^{2+}$ and the stronger protein-binding properties of $\mathrm{Ca}^{2+}$. Finally, it is important to remark that assuming that $\Gamma_{\mu_{2}}$ can be entirely described in terms of protein-salt partitioning at the surface of the macromolecule by neglecting the commonion effects (i.e., $\chi_{21} \approx-\Gamma_{\mu_{2}} / m_{2}$ ) may lead to qualitatively inaccurate interpretations of the experimental $\Gamma_{\mu_{2}}$.

\section{Appendix}

Two-Domain Model for Electrolytes with Polyvalent Cations in the Presence of Positively Charged Macromolecules. According to the two-domain model, partitioning of water molecules and ions between the protein layers and the bulk phase are described by the following three mass balances on the total number of molecules: $n_{\mathrm{M}}, n_{\mathrm{C}}$, and $n_{0}$ partitioning between the protein layer domains: $N_{\mathrm{M}}, N_{\mathrm{C}}$, and $N_{0}$, and the bulk phase: $n_{\mathrm{M}}^{(\mathrm{b})}, n_{\mathrm{C}}^{(\mathrm{b})}$, and $n_{0}^{(\mathrm{b})}$.

$$
\begin{aligned}
& \frac{n_{\mathrm{M}}}{n_{1}}=N_{\mathrm{M}}+\frac{n_{\mathrm{M}}^{(\mathrm{b})}}{n_{1}} \\
& \frac{n_{\mathrm{C}}}{n_{1}}=N_{C}+\frac{n_{\mathrm{C}}^{(\mathrm{b})}}{n_{1}} \\
& \frac{n_{0}}{n_{1}}=N_{0}+\frac{n_{0}^{(\mathrm{b})}}{n_{1}}
\end{aligned}
$$

Equation A3 can be solved with respect to $n_{1}$. By inserting the obtained expression of $n_{1}$ into the right side of eqs A1 and A2, we can obtain explicit equations for the bulk molalities of the ions $m_{\mathrm{M}}^{(\mathrm{b})}$ and $m_{\mathrm{C}}^{(\mathrm{b})}$,

$$
\begin{aligned}
& m_{\mathrm{M}}^{(\mathrm{b})}=\frac{m_{\mathrm{M}}-N_{\mathrm{M}} m_{1}}{1-N_{0}\left(m_{1} / m_{0}\right)} \\
& m_{\mathrm{C}}^{(\mathrm{b})}=\frac{m_{\mathrm{C}}-N_{\mathrm{C}} m_{1}}{1-N_{0}\left(m_{1} / m_{0}\right)}
\end{aligned}
$$

where we have substituted both the total and bulk molecular ratios with the corresponding molality ratios. The bulk domain, which is not assumed to be electroneutral, can be thought to be in Donnan equilibrium with a binary salt-water solution with molality $m_{2}^{(\mathrm{b})}$. If $z_{\mathrm{M}}$ is the charge of the salt cation, the molality of the binary salt-water solution is

$$
m_{2}^{(\mathrm{b})}=\left(\frac{m_{\mathrm{M}}^{(\mathrm{b})} m_{\mathrm{C}}^{(\mathrm{b})^{z \mathrm{M}}}}{z_{\mathrm{M}}^{z_{\mathrm{M}}}}\right)^{1 / z_{\mathrm{M}}+1}
$$

We can then replace $m_{\mathrm{M}}^{(\mathrm{b})}$ and $m_{\mathrm{C}}^{(\mathrm{b})}$ with $m_{2}^{(\mathrm{b})}$ by taking the product of eqs A4 and A5 according to eq A6. This yields

$$
z_{\mathrm{M}}{ }^{z_{\mathrm{M}}} m_{2}{ }^{(b){ }^{\left(z_{\mathrm{M}}+1\right)}}=\frac{\left(m_{\mathrm{M}}-N_{\mathrm{M}} m_{1}\right)\left(m_{\mathrm{C}}-N_{\mathrm{C}} m_{1}\right)^{z_{\mathrm{M}}}}{\left(1-N_{0} m_{1} / m_{0}\right)^{\left(z_{\mathrm{M}}+1\right)}}
$$

Since $m_{\mathrm{M}}=m_{2}$ and $m_{\mathrm{C}}=z_{\mathrm{M}} m_{2}+z_{\mathrm{P}} m_{1}$, eq A7 yields to first order in $m_{1}$.

$$
-\frac{m_{2}-m_{2}^{(\mathrm{b})}}{m_{1}}=\frac{z_{\mathrm{P}}}{z_{\mathrm{M}}+1}+N_{0} \frac{m_{2}^{(b)}}{m_{0}}-\frac{N_{\mathrm{M}}+N_{\mathrm{C}}}{z_{\mathrm{M}}+1}
$$

In the limit of $m_{1} \rightarrow 0, \Gamma_{\mu_{2}}=\left(m_{2}-m_{2}^{(\mathrm{b})}\right) / m_{1}$ and $m_{2}^{(\mathrm{b})}=m_{2}$. 
This finally yields

$$
-\Gamma_{\mu_{2}}=\frac{z_{\mathrm{P}}}{z_{\mathrm{M}}+1}+N_{0} \frac{m_{2}}{m_{0}}-\frac{N_{\mathrm{M}}+N_{\mathrm{C}}}{z_{\mathrm{M}}+1}
$$

Acknowledgment. We thank Donald G. Miller for insightful discussions. The support of the NASA Microgravity Biotechnology Program through Grant NAG8-1356 is gratefully acknowledged.

Supporting Information Available: Additional information as noted in text. This material is available free of charge via the Internet at http://pubs.acs.org.

\section{References and Notes} 7367.

(1) Timasheff, S. N. Proc. Natl. Acad. Sci.U.S.A. 1998, 95, 7363-

(2) Parsegian, V. A.; Rand, R. P.; Rau. D. C. Proc. Natl. Acad. Sci. U.S.A. 2000, 97, 3987-3992. 9726.

(4) Schellman, J. A. Q. Rev. Biophys. 2005, 38, 351-361.

(5) Wyman, J., Jr. Adv. Protein Chem. 1964, 19, 223-286.

(6) Retailleau, P.; Ducruix, A.; Riès-Kautt, M. Acta Crystallogr. 2002, D58, 1576-1581

(7) Courtneay, E. S.; Capp, M. W.; Anderson, C. F.; Record, M. T., Jr. Biochemistry 2000, 39, 4455-4471.

(8) Annunziata, O.; Asherie, N.; Lomakin, A.; Pande, J.; Ogun, O.; Benedek, G. B. Proc. Natl. Acad. Sci. U.S.A. 2002, 99, 14165-14170.

(9) Ru, M. T.; Hirokane, S. Y.; Lo, A. S.; Dordick, J. S.; Reimer, J. A.; Clark, D. S. J. Am. Chem. Soc. 2000, 122, 1565-1571.

(10) Annunziata, O.; Paduano, L.; Pearlstein, A. J.; Miller, D. G.; Albright, J. G. J. Am. Chem. Soc. 2000, 122, 5916-5928.

(11) Arakawa, T.; Bhat, R.; Timasheff, S. N. Biochemistry 1990, 29, 1914-1923. 794.
(13) Arakawa, T.; Timasheff, S. N. Biochemistry 1984, 23, 5912-5923.

(14) McPherson, A. Crystallization of Biological Macromolecules; Cold Spring Harbor: New York, 1998.

(15) Tanford, C. Physical Chemistry of Macromolecules; Wiley: New York, 1961.

(16) Warren, P. B. J. Phys.: Condens. Matter 2002, 14, 7617-7629.

(17) Annunziata, O.; Paduano, L.; Pearlstein, A. J.; Miller, D. G.; Albright, J. G. J. Phys. Chem. B 2006, 110, 1405-1415.

(18) Annunziata, O.; Paduano, L.; Albright, J. G. J. Phys. Chem. B 2006 $110,16139-16147$

(19) Albright, J. G.; Annunziata, O.; Miller, D. G.; Paduano, L.; Pearlstein, A. J. J. Am. Chem. Soc. 1999, 121, 3256-3266.

(20) Leaist, D. G. J. Phys. Chem. 1989, 93, 474-479.

(21) Gosting, L. J. Adv. Prot. Chem. 1956, 11, 429-554.

(22) We use the subscript "1" for the protein component and the subscript " 2 " for the salt component, consistent with the literature on multicomponent diffusion; however, the subscript "2" for the protein component and the subscript " 3 " for the salt component are extensively used in relation to the thermodynamic description of protein mixtures.

(23) Paduano, L.; Annunziata, O.; Pearlstein, A. J.; Miller, D. G.; Albright, J. G. J. Cryst. Growth 2001, 232, 273-284.

(24) Von Hippel, P. H.; Schleich, T. Acc. Chem. Res. 1969, 2, 257265 .

(25) Maguire, M. E.; Cowan, J. A. Biometals 2002, 15, 203-210.

(26) Rard, J. A.; Miller, D. G. J. Solution Chem. 1979, 8, 701-716.

(27) Annunziata, O.; Buzatu, D.; Albright, J. G. Langmuir 2005, 21 12085-12089.

(28) Kirkwood, J. G.; Baldwin, R. L.; Dunlop, P. J.; Gosting, L. J.; Kegeles, G. J. Chem. Phys. 1960, 33, 1505-1513.

(29) Dunlop, P. J.; Gosting, L. J. J. Phys. Chem. 1959, 63, 86-93.

(30) Miller, D. G.; Rard, A. J.; Eppstein, L. B.; Albright, J. G. J. Phys. Chem. 1984, 88, 5739-5748.

(31) Onsager, L. Phys. Rev. 1931, 38, 2265-2279.

(32) Woolf, L. A.; Miller, D. G.; Gosting, L. J. J. Am. Chem. Soc. 1962, $84,317-331$

(33) Miller, D. G.; Vitagliano, V.; Sartorio, R. J. Phys. Chem. 1986 90, 1509-1519.

(34) Miller, D. G. J. Phys. Chem 1959, 63, 570-578.

(35) Robinson, R. A.; Stokes, R. H. Electrolyte Solutions; Academic Press: New York, 1955.

(36) Isono, T. J. Chem. Eng. Data 1984, 29, 45-52.

(37) Grisby, J. J.; Blanch, H. W.; Prausnitz, J. M. J. Phys. Chem. B 2000, 104, 3645-3650.

(38) Tanford, C.; Wagner, M. L. J. Am. Chem. Soc. 1954, 76, 3331- 